\title{
Carbon ion beam combined with cisplatin effectively disrupts triple negative breast cancer stem-like cells in vitro
}

Sei Sai ${ }^{*}$, Guillaume Vares ${ }^{2}$, Eun Ho Kim ${ }^{3}$, Kumiko Karasawa ${ }^{4}$, Bing Wang ${ }^{2}$, Mitsuru Nenoi ${ }^{2}$, Yoshiya Horimoto ${ }^{5}$ and Mitsuhiro Hayashi ${ }^{6}$

\begin{abstract}
Aims: Although a relatively small proportion of all breast cancer (BC), triple negative (TN) BC is responsible for a relatively large proportion of $\mathrm{BC}$ deaths because of its worse clinical outcome. To investigate whether a carbon ion beam alone or in combination with cisplatin (CDDP) has a beneficial effect compared to X-rays, we target triple negative (TN) breast cancer stem-like cells (CSCS).

Methods: Human breast CSCs sorted from MDA-MB-231 and MDA-MB-453 cells were treated with a carbon ion beam or X-ray irradiation alone or in combination with CDDP, and then colony, spheroid and tumor formation assays, RT-PCR Array analysis, and immunofluorescence $\mathrm{H} 2 \mathrm{AX}$ foci assay were performed.

Results: The colony, spheroid formation, and tumorigenicity assays confirmed that CD44+/CD24- and ESA+/CD24- cells have CSC properties in MDA-MB-231 and MDA-MB-453 cells, respectively. The proportion of CSCs was more enriched after CDDP combination with either X-ray or carbon ion beam, however carbon ion beam combined with CDDP significantly suppressed colony and spheroid formation and more significantly inhibited cell cycle progression (sub-G1 arrest) compared to X-ray combined with CDDP or carbon ion beam alone. RT-PCR Array analysis showed that carbon ion beam combined with CDDP significantly induced apoptosis-related Cytochrome c, almost completely eliminated expression of the CSC markers CD44 and ESA, and significantly inhibited angiogenesis, and metastasis-related HIF1 $a$ and CD26 compared to carbon ion beam alone, X-ray alone, or X-ray combined with CDDP. The immunofluorescence assay showed that not only the number but also the size of $Y H 2 A X$ foci in CSCs were larger $24 \mathrm{~h}$ after carbon ion beam combined with CDDP compared to those of $\mathrm{X}$-ray alone and $\mathrm{X}$-ray combined with CDDP.
\end{abstract}

Conclusions: Carbon ion beam combined with CDDP has superior potential to kill TN breast CSCs with irreparable severe DNA damage and enhanced apoptosis.

Keywords: Heavy-ion radiation, Breast cancer stem cell, Cisplatin

\section{Introduction}

Human breast cancer (BC) has become one of the leading causes of cancer-related death for women worldwide, and it is rapidly increasing in Asian countries including Japan [1-3]. BC represents a group of highly heterogeneous lesions consisting of morphologically distinct

\footnotetext{
*Correspondence: saisei@nirs.go.jp

'Medical Physics Research Program, Research Center for Charged Particle Therapy, National Institute of Radiological Sciences, 4-9-1 Anagawa Inage-ku, Chiba, Chiba 263-8555, Japan

Full list of author information is available at the end of the article
}

subtypes [4], with different molecular and biochemical signatures [5].

Triple-negative breast cancers (TNBC), defined as tumors that are negative for estrogen receptor (ER), progesterone receptor (PR) and human epidermal growth factor receptor 2 (HER2), nowadays represent the focus of increasing interest at the clinical, biological and epidemiological level [6-8], due to the aggressive behavior of the tumor, poor prognosis and present lack of targeted therapies [9-11]. According to current estimates, TNBC accounts for $10-17 \%$ of all $\mathrm{BC}$, depending on

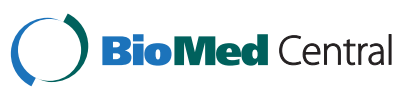


thresholds used to define ER and PR positivity and HER2 overexpression $[12,13]$. Despite its relatively small proportion among all $\mathrm{BCs}$, TNBC is responsible for a relatively large proportion of $\mathrm{BC}$ deaths, due to its generally aggressive clinical course.

The very high rate of heterogeneity in $\mathrm{BC}$ cell phenotypes [14], accompanied by the dynamic plasticity of the $\mathrm{BC}$ microenvironment [15], make tumor categorization a demanding task, especially in relation to therapeutic responses and risk of disease progression [16]. Breast cancer stem-like cell (BCSC) populations have recently been identified based on the cell membrane markers CD44+/CD24-/ ESA+ cells $[17,18]$. CSCs represent the tumor's subpopulation with the highest capacity to drive its growth, invasion and metastasis. BCSCs are endowed with the capacity for self-renewal and multi-lineage differentiation, tumorigenicity, and chemotherapy and radiotherapy resistance, features that are responsible for tumor progression, disease recurrence, and metastasis [19-21]. It has been reported that CSC subpopulations are relatively radioresistant compared with non-CSC subpopulations, because of their high DNA repair capability and upregulated survival pathways that protect them from various cellular stresses including radiation. Thus, the development of new potent CSC targeting therapeutics is highly desirable [22-24].

The heavy ion medical accelerator in Chiba (HIMAC) at the National Institute of Radiologic Science (NIRS) has treated more than 9000 patients with various radioresistant tumors, and achieved promising results to date [25-27]. The heavy ion beams have a well-defined range and insignificant scatter in tissues with well-localized energy deposition at the end of the beam path, called the "spread out bragg peak (SOBP)", a unique physical characteristic of charged particle beams, and release enormous energy at the end of their range. They therefore induce more cell cycle- and oxygenation-independent, irreparable DNA damage and kill more resistant cancer cells than conventional radiation [28, 29]. Recently, a phase I clinical trial of early stage $\mathrm{BC}$ treatment with heavy ion radiotherapy was started. However, because of limitations of dose elevation because of side effects on skin, ribs, and lungs after carbon ion radiotherapy, especially for some aggressive subtypes of BC like TNBC, we thought that carbon ion beam combined with chemotherapy may reduce the doses of irradiation but still have some advantage to destroy $\mathrm{BC}$. The combination of chemotherapy with heavy ion radiotherapy may open new perspectives in the fight against this challenging $\mathrm{BC}$ subgroup with worse prognosis and still limited therapy options.

Recently, we have reported that BCSCs can be generated by steroid hormones in irradiated breast cell lines [30], and also shown that a carbon ion beam has a marked effect on colon and pancreatic CSCs, which are resistant to photon beams [31, 32]. Considering the fact that cisplatin (CDDP) has been reported to be effective in treating TNBC [33], in the present study, we try to examine the effects of a carbon ion beam alone or in combination with CDDP on putative BCSCs survival, DNA repair, and expression changes of various genes compared to that of X-ray irradiation. To the best of our knowledge, this is the first study to show heavy ion radiation combined with CDDP has an advantage in targeting BCSCs at relatively low doses compared to carbon ion beam alone or conventional X-ray irradiation.

\section{Materials and methods}

\section{Cell lines and reagents}

Human triple negative breast cancer cell lines, MDAMB-231 and MDA-MB-453 were purchased from American Type Culture Collection (Manassas, VA). Unsorted cells were cultured in Dulbecco's Modified Eagle's medium (DMEM) supplemented with $10 \%$ heat-inactivated fetal bovine serum (Beit-HaEmek, Israel), $100 \mathrm{unit} / \mathrm{mL}$ penicillin and $100 \mu \mathrm{g} / \mathrm{mL}$ streptomycin (Invitrogen) at $37{ }^{\circ} \mathrm{C}$ with $5 \% \mathrm{CO}$-in-air. The medium was changed every other day. CSCs and non-CSCs isolated from MDA-MB-231 and MDA-MB-453 cells were cultured with serum-free Essential 8 medium (Life technologies Japan Ltd, Tokyo). CDDP was purchased from Sigma Japan. The CDDP solutions were diluted in PBS immediately before use.

\section{Animals}

NOD/SCID mice (6-8 weeks old, Charles River Laboratories, Yokohama, Japan) were maintained under defined conditions at the NIRS animal facility. The animals were observed at least 24 weeks, and tumorigenicity was determined when tumor nodules were identified on their body surfaces. Tumor formation assay for MDAMB-231 delivered CD44+/CD24- and CD44-/CD24- cells and MDA-MB-453 delivered ESA+/CD24- and ESA-/ CD24+ cells were also performed as described previously [32]. All experiments involving the use of animals were performed in accordance with NIRS institutional animal welfare guidelines.

\section{Colony and spheroid formation assays}

Clonogenic survival assay was performed as described previously [31, 32]. In brief, the appropriate plating density was aimed at producing 20-40 surviving colonies in each T-25 flask. After incubation for 14 days, the colonies were fixed and stained with $0.3 \%$ methylene blue in ethanol, and colonies containing more than 50 cells were counted as survivors. At least three parallel samples were scored in three to five repetitions performed for each type of irradiation. Clonogenicity 
and/or spheroid formation ability assay for CD44+/ CD24- and CD44-/CD24- cells sorted from MDA-MB-231 cells and ESA-/CD24+ and ESA+/CD24- cells sorted from MDA-MB-453 cells were performed as described previously [31]. The data is presented as the percentage of the wells that contained spheres, and the average size using WinRoof 5.6 software (Mitani Corporation, Tokyo, Japan) after 1-week incubation.

\section{Irradiation}

Cells were irradiated with carbon-ion beams (accelerated by the HIMAC). Briefly, the initial energy of the carbonion beams was $290 \mathrm{MeV} / \mathrm{n}, 50 \mathrm{KeV} / \mu \mathrm{m}$, center of $6 \mathrm{~cm}$ Spread-Out Bragg Peak (SOBP). As a reference, cells were also irradiated with conventional $200 \mathrm{kVp}$ X-ray (TITAN-320, GE Co., USA).

\section{FACS analysis}

FACS analysis for the cells irradiated with X-rays or carbon ion beams was performed with BD FACS Aria (Becton Dickinson, San Jose, CA, USA) as described previously [31]. In brief, the cells were prepared and labeled with conjugated anti-human CD44-PE (Miltenyi Biotec), ESA-APC (Miltenyi Biotec), and CD24-FITC. Isotype matched immunoglobulin served as control. Cells were incubated for $20 \mathrm{~min}$ at each step and were washed with $2 \%$ FBS/PBS between steps. The percentage of CD44+, ESA+, and $\mathrm{CD} 24+$ present was assessed after correction for the percentage of cells reactive with an isotype control.

\section{Cell cycle analysis}

After harvesting and washing cells with PBS, they were fixed in ice-cold $70 \%$ ethanol (ethanol in distilled water) while vortexing, then stained with propidium iodide $(1 \mu \mathrm{g} / \mathrm{mL}$, Sigma) in the presence of RNase A, and then analyzed using a BD FACS Calibur flow cytometer (BD Biosciences). A minimum of 10,000 cells were counted for each sample, and data analysis was performed with CellQuest software.

\section{PCR Profiler array analysis of various gene expression} related to apoptosis, autophagy and DNA repair

The Human Custom RT $^{2}$ Profiler ${ }^{\mathrm{ru}}$ PCR Array (CAPH11870A, Qiagen) profiles the expression of 42 genes involved in DNA damage, apoptosis, autophagy, and angiogenesis. RNA was purified using the Qiagen RNAeasy kit, including on-column DNAse treatment to remove genomic DNA. cDNA was prepared with the $\mathrm{RT}^{2}$ First Strand Kit (SABiosciences, Frederick, Maryland, USA). A PCR profiler array specific for $48 \times 2$ OSRGs was performed $\left(\mathrm{RT}^{2}\right.$ SYBR Green/ROX qPCR Master Mix; SABiosciences) in 96-well microtiter plates on an ABI 7300 instrument (Applied Biosystems, California, USA). For data analysis, the $\Delta \Delta \mathrm{Ct}$ method was applied using the $\mathrm{RT}^{2}$ Profiler PCR Array software package and statistical analyses performed $(n=3)$. This package uses $\Delta \Delta \mathrm{C}_{\mathrm{T}}$-based fold change calculations and the Student's $t$-test to calculate two-tail, equal variance $\mathrm{p}$-values. The fold changes were calculated using the equation $2^{-\Delta \Delta C t}$. If fold change was greater than 1 , the result was considered as fold-upregulation. If fold change was less than 1 , the negative inverse of the result was considered as folddownregulation [34].

\section{$\mathrm{\gamma H} 2 \mathrm{AX}$ Immunofluorescence assay}

$\gamma \mathrm{H} 2 \mathrm{AX}$ Immunofluorescence assay was performed as described previously [32]. In brief, cultured cells grown on plastic chamber slides (Lab-Tek. Nunc, USA) were fixed in $4 \%$ formaldehyde for $15 \mathrm{~min}$ at room temperature. Then the cells were permeabilized in $0.2 \%$ Triton X-100 and blocked with $10 \%$ goat serum, then incubated with mouse monoclonal anti-phosphoHistone $\mathrm{H} 2 \mathrm{AX}(\mathrm{Ser} 139)(\gamma \mathrm{H} 2 \mathrm{AX})$ at $37{ }^{\circ} \mathrm{C}$ in PBS with $10 \%$ goat serum and washed with PBS. The cells were incubated with the Alexa 488 anti rabbit secondary antibody at $37^{\circ} \mathrm{C}$ in PBS with $10 \%$ goat serum and washed in PBS. Cover glasses were mounted in ProLong ${ }^{\circ}$ Gold antifade reagent with DAPI (Invitrogen). Fluorescence images were captured using an Olympus DP70 fluorescence microscope for analysis. All treatment groups were then assessed for $\gamma \mathrm{H} 2 \mathrm{AX}$ foci via sequential imaging through each nucleus. A minimum of 100 cells in each treatment group were counted. Nuclear $\gamma \mathrm{H} 2 \mathrm{AX}$ foci size was estimated by WinRoof 5.6 software (Mitani Corporation, Tokyo, Japan) .

\section{Statistical analysis}

One-way analysis of variance (ANOVA) and Bonferroni multiple comparison tests were used when mean differences between the groups were evaluated by StatView software (SAS Institute, Inc., Cary, NC). For all comparisons, $p$ values less than 0.05 were defined as significant.

\section{Results \\ Determination of cancer stem-like cell properties of CD44+/CD24- and ESA+/CD24- cells sorted from MDA-MB-231 and MDA-MB-453 cells}

As shown in Fig. 1, CD44+/CD24- cells had greater colony and sphere formation abilities than CD44+/CD24cells. When an equal number of 5000 cells were plated in a dish, CD44+/CD24- cells from MDA-MB-231 formed $135+6$ clones, whereas CD44+/CD24- cells formed only $78+2$ clones $(p<0.01)$. These data showed that CD44+/CD24- BC cells had much greater clonal formation capacities than those of CD44-/CD24- cells (Fig. 1a). To investigate the ability to form spheroid bodies, isolated CD44+/CD24- and CD44-/CD24- cells were cultured in 96-well round-bottomed Sumilon celltight 


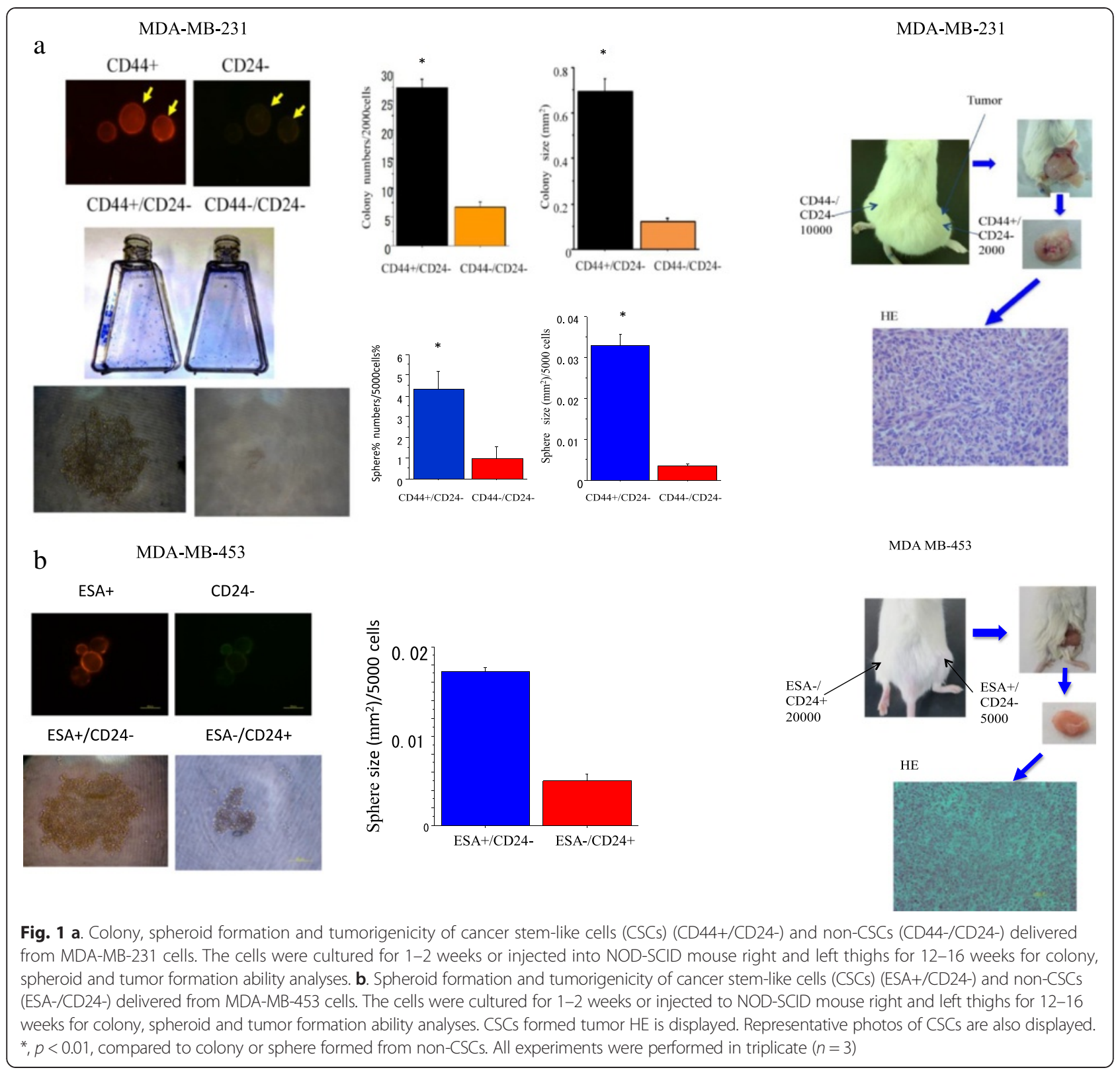

spheroid plates (Sumilon, Sumitomo Bakelite Co., Tokyo, Japan). After being in culture for 1 week, the ability of CD44+/CD24- cells to form spheroid bodies was significantly higher both in number and in size than that of CD44-/CD24- (p < 0.01) (Fig. 1a). CD44 was almost undetectable but the ESA was detectable from MDA-MB-453 cells. As shown in Fig. 1b, the spheroid formation ability of ESA+/CD24- is significantly higher than ESA-/CD24+ cells sorted from MDA-MB-453 cells.

To examine in vivo tumorgenicity, various numbers of CD44+/CD24- or CD44-/CD24- cells isolated from MDA-MB-231, and ESA+/CD24- or ESA-/CD24+ isolated from MDA-MB-453 cells were subcutaneously transplanted into the right or left lower thigh of immunodeficient NOD SCID mice. As shown in Fig. 1 and Additional file 1: Table S1, only 5000 cells of CD44+/CD24-/ cells could form a tumor whereas $1 \times$ $10^{4}$ CD44-/CD24- or ESA-/CD24+ cells could not, suggesting that CD44+/CD24-and ESA+/CD24- cells have characteristics of cancer stem-like cells.

\section{Changes in proportion of CD44+/CD24- and ESA+/CD24- cells after carbon-ion beam alone or in combination with CDDP}

The percentage changes of cancer stem like CD44+/CD24cells in MDA-MB-231 cells and ESA+/CD24- cells in MDA-MB-453 cells $72 \mathrm{~h}$ or $96 \mathrm{~h}$ after carbon ion beam, X-ray alone or in combination with $25 \mu \mathrm{M}$ of 
CDDP were investigated by FACS analysis. As shown in Fig. 2a, the proportion of CD44+/CD24- cells was dose dependently increased after X-ray irradiation, whereas no significant changes by carbon ion beam at which the doses induced equivalent effects by X-ray. The percentage of CD44+/CD24- cells was increased more significantly when X-ray combined with CDDP compared to that of carbon ion beam with CDDP alone. However, the proportion of ESA+/CD24- cells in MDA-MB-453 cells was decreased either by X-ray or carbon ion beam alone, but significantly increased by combination with CDDP or with CDDP alone (Fig. 2b).

\section{Surviving fraction of unsorted MDA-MB-231 cells and} sorted CSCs and non-CSCs after carbon ion beam or X-ray irradiation

The MDA-MB-231 cells were irradiated with X-ray or carbon ion beams and their surviving fraction was estimated by colony assay. The surviving fractions for the MDA-MB-231 cells irradiated with X-ray or carbon ion beam decreased exponentially with increasing doses. Based on the survival curve, the D10 (dose required to reduce the surviving fraction to $10 \%$ ) was estimated as 3.9 Gy for X-ray and 2.0 Gy for carbon ion beam. Therefore the relative biological effectiveness (RBE) values for SOBP carbon ion beams relative to X-rays at $\mathrm{D} 10$ level is about 1.80 (Fig. 3a).

The surviving fractions for the cancer stem-like CD44+/CD24- and non-cancer stem like CD44-/ CD24- cells sorted from MDA-MB-231 irradiated with $\mathrm{X}$-rays and carbon ion beams decreased exponentially with increasing doses, and CD44-/CD24- cells more significantly decreased compared to that of CD44+/CD24cells after irradiation with either X-rays or carbon ion beams (Fig. 3a). The RBE values calculated at the D10 level for CSCs were calculated to be about 2.14, whereas

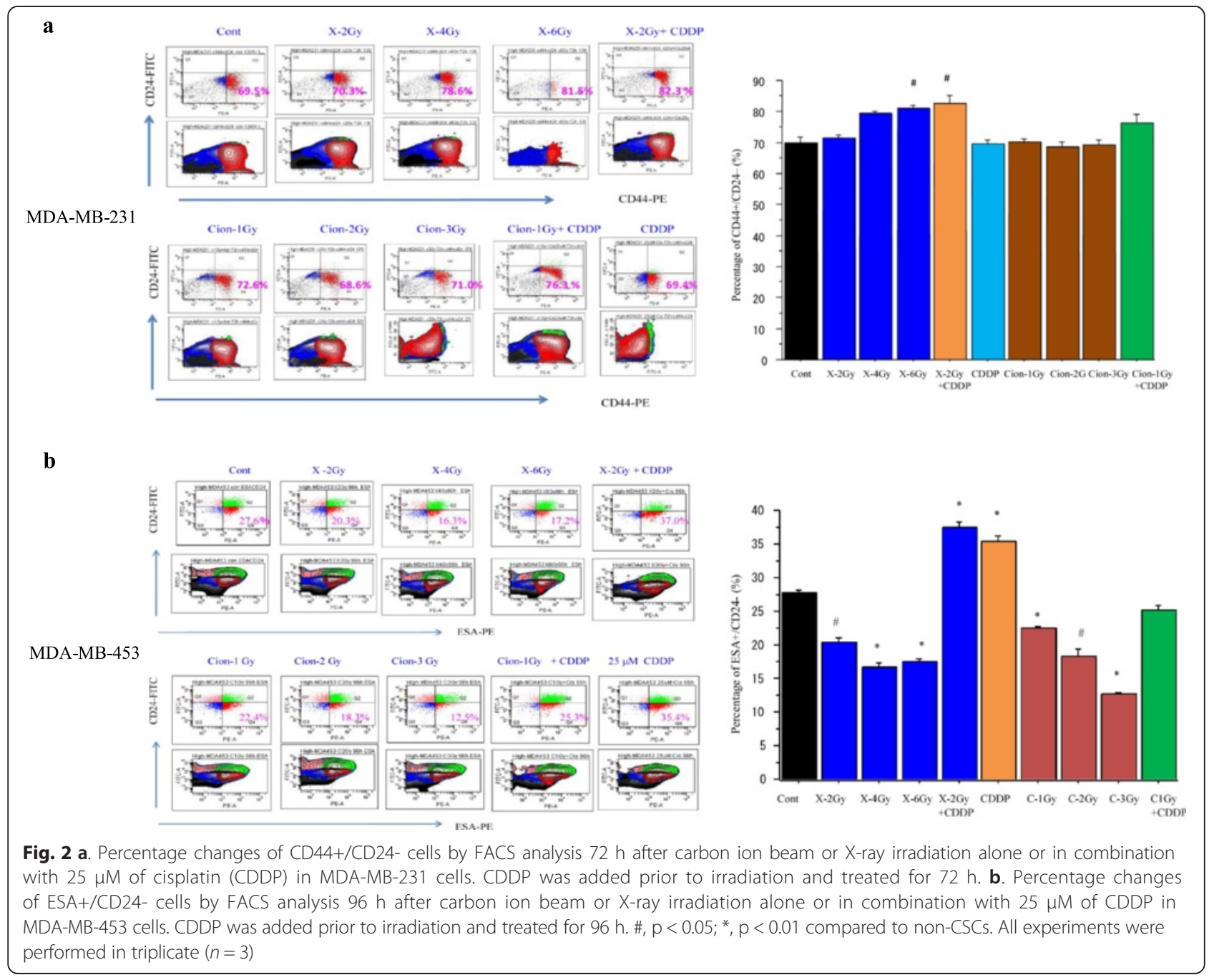




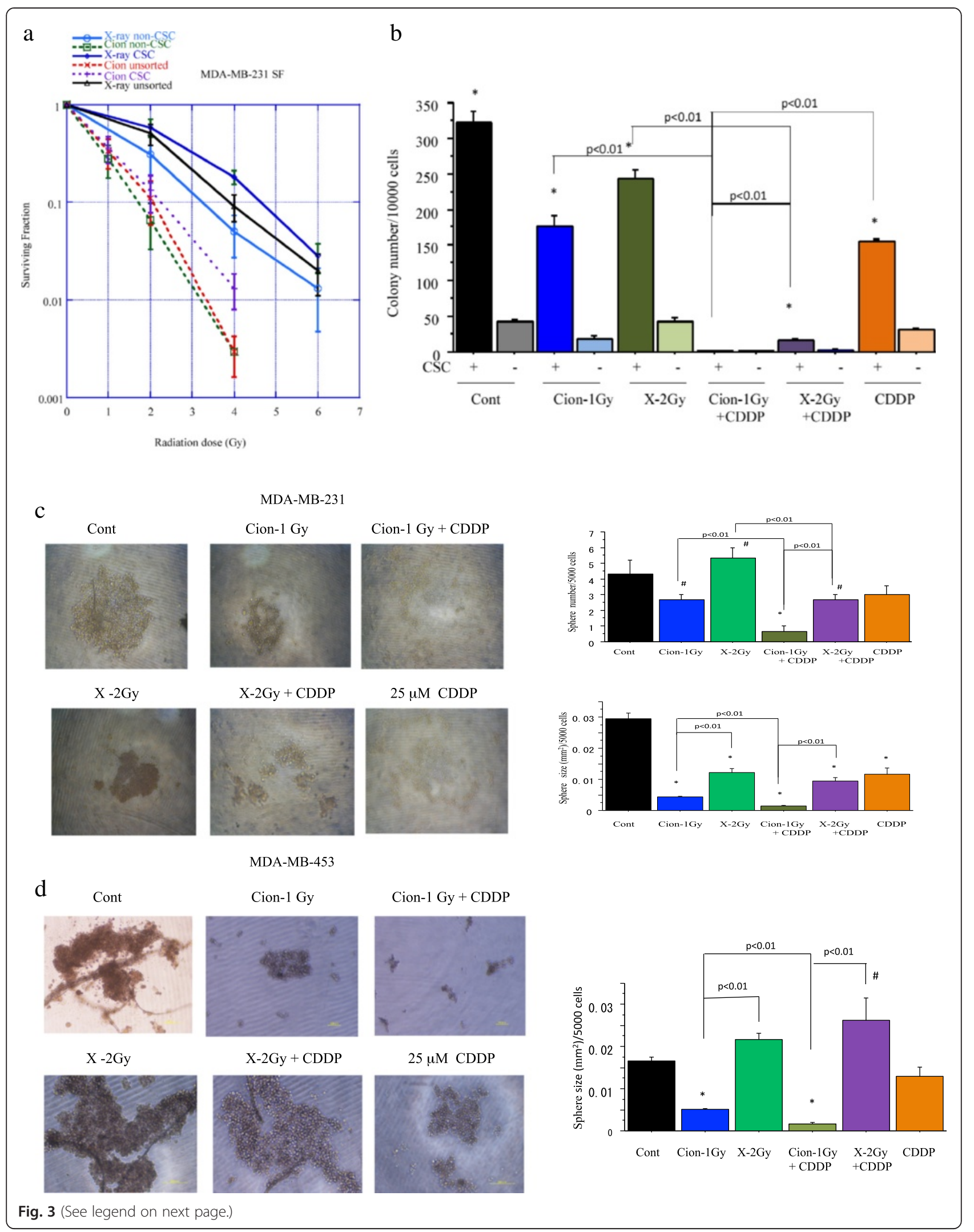


(See figure on previous page.)

Fig. 3 a. Surviving fraction of unsorted MDA-MB-231 cells and CSCS (CD44+/CD24-) and non-CSCs (CD44-/CD24-) delivered from MDA-MB-231 cells plated immediately after carbon ion beam or X-ray irradiation. The graphs show the mean and standard error calculated from three independent experiments. All experiments were performed in triplicate $(n=3)$. b. Quantification of colony formation of $(C D 44+/ C D 24-)$ and non-CSCs (CD44-/CD24-) after X-ray, a carbon ion beam alone or in combination with $25 \mu \mathrm{M}$ of CDDP. CDDP was added prior to irradiation and treated for 5 days. ${ }^{*}, p<0.01$, compared to non-CSCs. Representative photos and quantification of spheroid size formed from MDA-MB-231 delivered CSCs (CD44+/CD24-) (c) and MDA-MB-453 delivered CSCs (ESA+/CD24-) (d) after X-ray, a carbon ion beam alone or in combination with $25 \mu \mathrm{M}$ of CDDP. The spheroid formation was observed 7 days after X-ray, a carbon ion beam alone or in combination with CDDP. CDDP was added prior to irradiation and treated for 5 days. The graphs show the mean and standard error calculated from three independent experiments. ${ }^{*}, p<0.01$, compared to non-CSCs. All experiments were performed in triplicate $(n=3)$

those for non-CSCs were about 1.78. RBE values for unsorted and sorted CSCs and non-CSCs of carbon ion beams relative to X-rays are summarized in Table 1.

Colony and/or spheroid formation ability of CD44+/CD24and ESA+/CD24- cells sorted from MDA-MB-231 and MDA-MB-453 cells after carbon-ion beam or X-ray alone or in combination with CDDP

To examine the effects of cisplatin on radiosensitization to X-rays and carbon ion beams, colony as well as spheroid formation ability of cancer stem-like CD44+/CD24cells and non-cancer stem-like CD44-/CD24- cells after irradiation with an X-ray or carbon ion beam alone or in combination with CDDP were performed. We found that the number of colonies from both CSCs and nonCSCs was remarkably reduced when carbon ion beam combined with $25 \mu \mathrm{M}$ of CDDP compared to carbon ion beam alone or X-ray combined with CDDP (Fig. 3b). As shown in Fig. 3c, the number of tumor spheroid formations of cancer stem like CD44+/CD24- cells delivered from MDA-MB-231 cells was significantly reduced after carbon ion beam compared to X-ray irradiation, and it was extremely decreased when the carbon ion beam combined with CDDP. In contrast, there are no spheres formed in non-cancer stem-like CD44-/CD24- cells after $\mathrm{X}$-ray or carbon ion beam, either alone or in combination with CDDP. Spheroid formation ability of ESA+/CD24- cells sorted from MDA-MB-453 after irradiation with an X-ray or carbon ion beam alone or in combination with CDDP was also performed, and showed the carbon ion beam alone but not X-ray significantly inhibited spheroid size and it was remarkably suppressed when carbon ion beam combined with CDDP (Fig. 3d).

Table 1 RBE values at D10 level for unsorted MDA-MB-231 cells and sorted cancer stem-like and non-cancer stem-like cells

\begin{tabular}{llll}
\hline Cells & X-ray & C-ion & RBE \\
\hline MDA-MB-231 & $3.9 \pm 0.11 \mathrm{~Gy}$ & $2.0 \pm 0.05 \mathrm{~Gy}$ & 1.80 \\
unsorted & $4.52 \pm 0.12 \mathrm{~Gy}$ & $2.12 \pm 0.11 \mathrm{~Gy}$ & 2.14 \\
CD44+/CD24- & $3.21 \pm 0.11 \mathrm{~Gy}$ & $1.82 \pm 0.06 \mathrm{~Gy}$ & 1.78 \\
\hline
\end{tabular}

\section{Expression changes of various genes in CSCs after} carbon-ion beam alone or in combination with CDDP by RT PCR Array analysis

To quantitatively examine multiple gene expression changes in radioresistant CSCs (CD44+/CD24-) delivered from MDA-MB-231 cells, $\mathrm{RT}^{2}$ Profiler PCR Array analysis was performed according to the manufacture's protocol. A representative clusterhistogram is shown in Fig. 4a. The data shows that treatment with a carbon ion beam combined with constant treatment with $25 \mu \mathrm{M}$ of CDDP for 5 days significantly increased the expressions of apoptosisrelated Cytochrome c, and had a strong tendency to increment $\operatorname{Bax}(p=0.059)$ and autophagy-related genes LC3 $(p=0.057)$ compared to carbon ion beam, X-ray, cisplatin alone or X-ray combined with CDDP (Fig. 4b). In addition, expressions of CSC markers, CD44 and ESA were almost completely eliminated by carbon ion beam combined with CDDP, whereas X-ray, carbon ion beam, CDDP alone or X-ray combined with CDDP (Fig. 4c) significantly increased expression of ESA. Besides, expressions of angiogenesis- and metastasis-related genes such as HIF $1 \alpha$ and CD26 were remarkably inhibited or lost by carbon ion beam combined with CDDP, whereas cisplatin alone or $\mathrm{X}$-ray combined with CDDP significantly increased expressions of HIF1 $\alpha$ and CD26 (Fig. 4d). Interestingly, some DNA repair-related genes such as XPC, Artemis, Rad51, and cell cycle-related gene PTEN were remarkably elevated but some of them, like XRCC4, 53BP1, and BRCA1, p27, and RB1 were significantly reduced by carbon ion beam combined with CDDP compared to carbon ion beam, $\mathrm{X}$-ray, CDDP alone or X-ray combined with CDDP (Fig. 4e, f).

Cell cycle analyses of MDA-MB-231 and MDA-MB-453 cells after carbon-ion beam alone or in combination with CDDP Cell cycle analyses of MDA-MB-231 and MDA-MB-453 cells 4 days after a carbon ion beam, X-ray alone or in combination with $25 \mu \mathrm{M}$ of CDDP were performed. CDDP was added prior to irradiation and constantly treated for 4 days, and the cell cycle distribution (sub G1, G1, S and G2/M phase) was measured by FACS Calibur. As shown in Fig. 5, carbon ion beam combined with CDDP more significantly inhibited cell cycle progression (sub-G1arrest) and induced cell death 


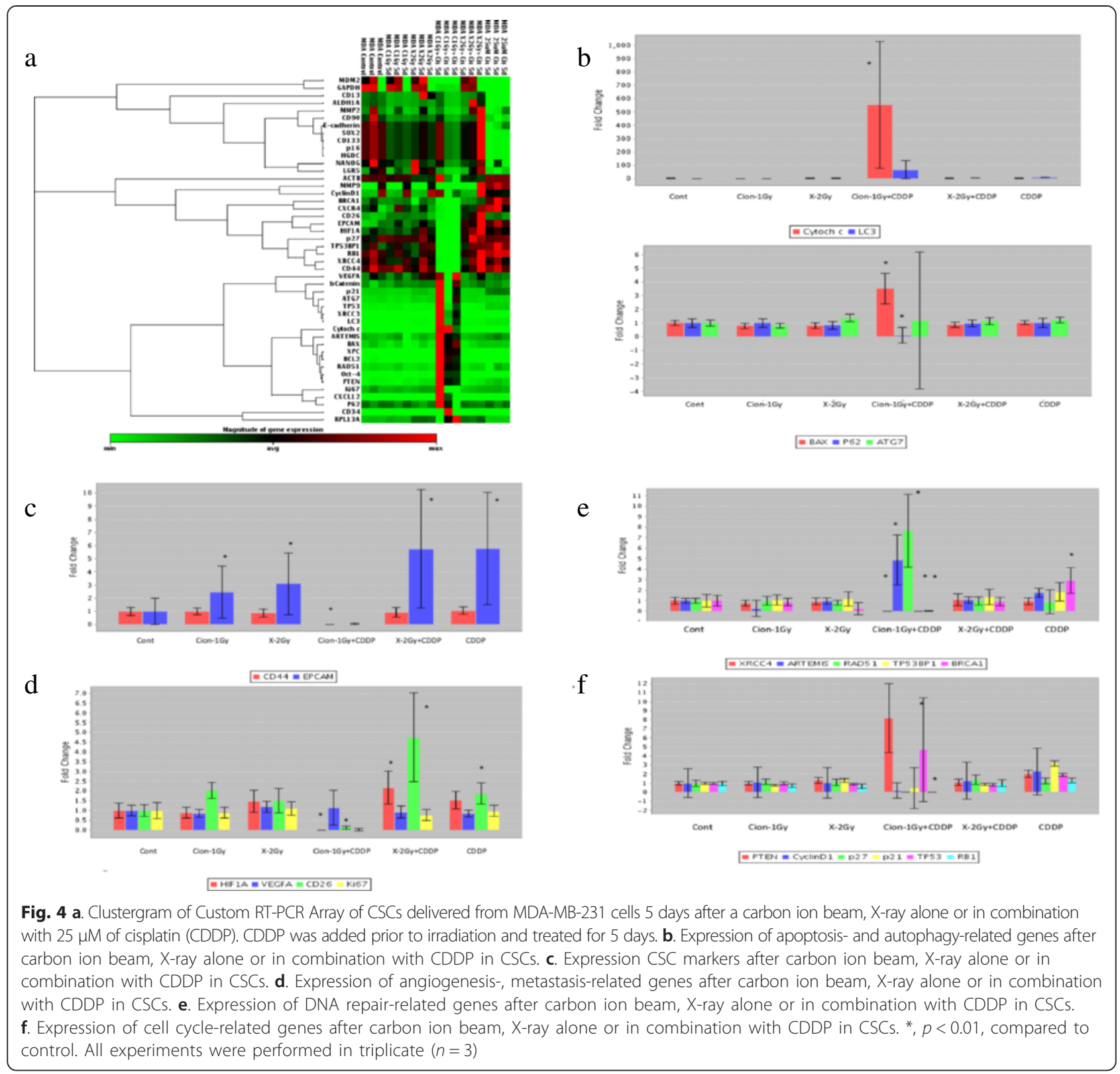

(apoptosis/necrosis) compared to carbon ion beam, X-ray alone or X-ray combined with CDDP both in MDA-MB231 and MDA-MB-453 cells.

\section{үH2AX foci formation in CD44+/CD24- and ESA+/CD24- cells after carbon-ion beam alone or in combination with CDDP}

A high number of $\gamma \mathrm{H} 2 \mathrm{AX}$ foci formed at $1 \mathrm{~h}$ after a carbon ion beam, X-ray alone, and in combination with $25 \mu \mathrm{M}$ of CDDP further increased the number of $\gamma \mathrm{H} 2 \mathrm{AX}$ foci in CD44+/CD24- cells sorted from MDAMB-231 (Fig. 5a). However, at $24 \mathrm{~h}$ after carbon ion beam irradiation, the induced $\gamma \mathrm{H} 2 \mathrm{AX}$ foci level remained significantly higher than that of X-ray irradiated cells with isoeffective dosages, and carbon ion beam in combination with cisplatin remarkably enhanced the number of $\gamma \mathrm{H} 2 \mathrm{AX}$ foci compared to carbon ion beam, X-ray, cisplatin alone or X-ray combined with cisplatin (Fig. 5a). Furthermore, not only a great increase in the number but also in the size of foci (clustered DSB) was frequently found in carbon ion beam combined with cisplatin-treated cells compared to carbon ion beam, X-ray, cisplatin alone or X-ray combined with cisplatin-treated cells (Fig. 5b). We also examined the number and size of nuclear $\gamma \mathrm{H} 2 \mathrm{AX}$ foci formed in CSCs (ESA+/CD24-) delivered from MDA-MB-453 cells at $24 \mathrm{~h}$ after a carbon ion beam, X-ray alone or in combination with $25 \mu \mathrm{M}$ of CDDP, and found that 


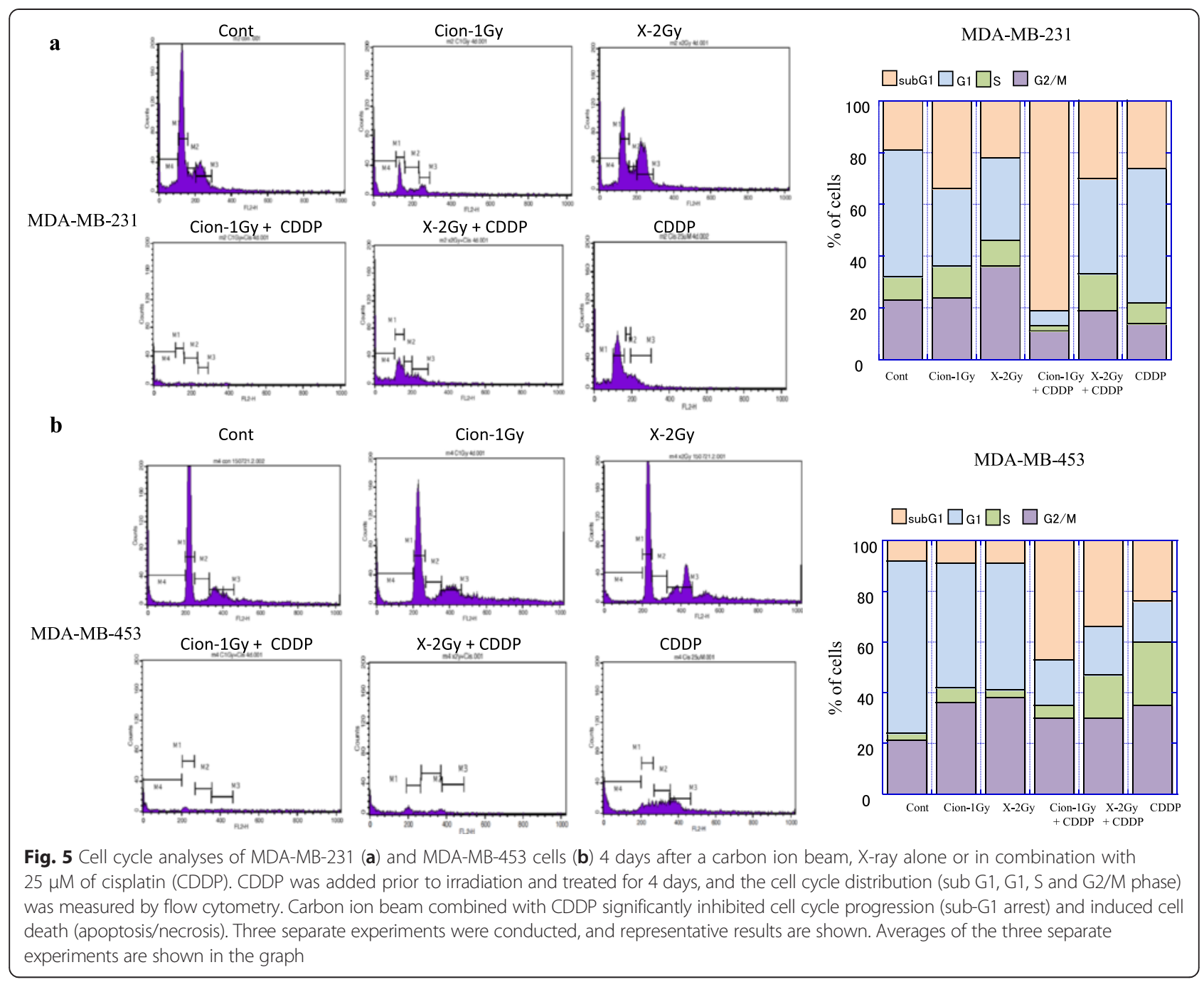

a much greater number of $\gamma \mathrm{H} 2 \mathrm{AX}$ foci were remained after carbon ion beam combined with CDDP, but surprisingly more larger sized $\gamma \mathrm{H} 2 \mathrm{AX}$ foci were induced by carbon ion beam alone compared to X-ray alone or in combination with CDDP (Fig. 6c).

\section{Discussion}

In this study, an in vitro colony and spheroid formation analysis as well as an in vivo tumorigenicity study showed that CD44+/CD24- cells have a significantly higher possibility compared to CD44-/CD24- cells which sorted from MDA-MB-231 cells, indicating that CD44+/CD24- cells exactly have CSC properties. We also examined and confirmed that ESA+/CD24- cells have CSC properties compared to ESA-/CD24+ which sorted from MDA-MB-453 cells based on its high spheroid formation and in vivo tumor formation ability.

This is in line with previous reports that CD44+/CD24and/or ESA+ /CD24- cells are BCSC markers [19, 35, 36]. We also investigated the proportion of ALDHhi, a typical
BCSC marker, is very low in both MDA-MB-231 and MDA-MB-453 cells (Additional file 2: Figure S1), so the CD44+/CD24- and ESA+/CD24- cells were representatively used as CSCs in this study.

We found that the percentages of cancer stem-like CD44+/CD24- cells in MDA-MB-231 cells were dosedependently increased after $72 \mathrm{~h}$ X-ray irradiation, whereas no such clear dose-response was observed after carbon ion beam (Fig. 2). In contrast, the proportion of $\mathrm{ESA}+/ \mathrm{CD} 24-$ cells in MDA-MB-453 cells significantly decreased at $96 \mathrm{~h}$ after carbon ion beam alone and surprisingly also by X-ray irradiation alone, but significantly increased by X-ray combined with CDDP or CDDP alone. The finding of CD44+/CD24- cells in MDA-MB231 cells is consistent with our and other previous reports [31,32,37], but it is unclear why the proportion of ESA+/CD24- cells in MDA-MB-453 cells was suppressed by $\mathrm{X}$-ray irradiation in this study. In the present study, the in vitro relative biological effectiveness (RBE) value calculated by the $\mathrm{D} 10$ relative to the $\mathrm{X}$-ray is about 


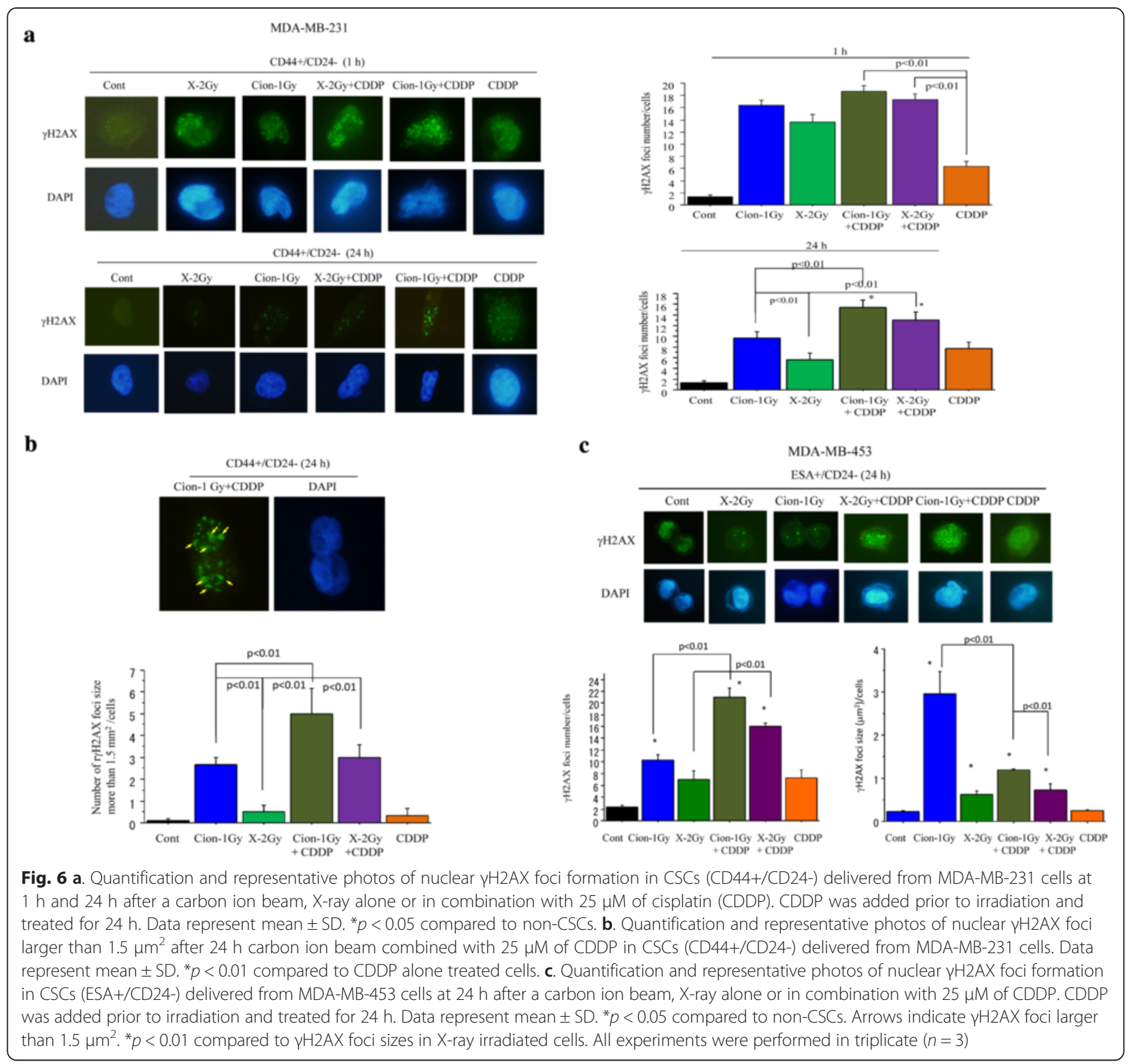

-1.75 to 1.85 for the center of SOBP carbon ion beam on MDA-MB-231 cells. RBE values are known to be dependent on linear transfer energy (LET), and our results are consistent with previous reports using carbon ion beams on several human cancer cells, which reported 1.57-2.60 for 50-80 keV/ $\mathrm{mm}$-beams [38]. Based on dose-response curves for cell-killing effect on CSCs and non-CSCs after irradiation with either X-rays or carbon ion beams, the CSCs showed resistance to both $\mathrm{X}$-rays and carbon ions compared to non-CSCs. The RBE values calculated at the D10 level for CSCs delivered from MDA-MB-231 were about 2.14, suggesting that the carbon ion beam has more power to destroy CSCs. In contrast, RBE values at the D10 level for non-CSCs delivered from MDA-MB-231 were only 1.78, implying that the difference in killing breast cancer cells between carbon ion beam and X-ray irradiation might mainly result from the strong effects on CSCs (Fig. 3a). Furthermore, the data shows that carbon ion beam combined with CDDP significantly decreased the number of colonies and the size of spheroids formed from MDA-MB231 and MDA-MB-453 delivered CSCs compared to Xray, carbon ion beam, CDDP alone or X-ray combined with CDDP, indicating that BCSCs were significantly radiosensitized when carbon ion beam was combined with CDDP (Fig. 3b, c, d).

In general, it has been suggested that CSC subpopulations are relatively radioresistant compared with nonCSC subpopulations, because of enhanced DNA repair capability with an increased ability to activate DNA 
damage checkpoint responses following radiation (e.g., activation of Chk1 and Chk2 checkpoint kinases), which serves to slow cell cycle progression and permit repair prior to cell division; quiescent cell cycle status (G0), hypoxic environment and upregulated survival pathways that protect from cellular stress [39]. It has been reported that CDDP radiosensitize breast cancer cells are accompanied with apoptosis and autophagy [40, 41]. In the present study, we found that after treatment with carbon ion beam in combination with CDDP for radioresistant CSCs delivered from MDA-MB-231 cells, not only apoptosis-related gene expressions like Cytochrome c but also autophagy-related genes like LC3 showed significant enhancement or a strong tendency to increase compared to that of carbon ion beam, X-ray and CDDP alone or X-ray combined with CDDP suggesting that carbon ion beam combined with CDDP may have more power to induce multiple cell death (Fig. 4a, b). It has been shown that CSCs are closely associated with radioresistance $[42,43]$. In this study, carbon ion beam combined with CDDP almost completely inhibited expression of CD44 and ESA. In contrast, carbon ion beam, X-ray, CDDP alone or X-ray combined with CDDP elevated ESA expression, suggesting that a relatively long-term treatment ( 5 days) by carbon ion beam combined with CDDP may have strong potential to eradicate BCSCs (Fig. 4c). It has been reported that CDDP can induce differentiation of CSC subpopulations within BC cell lines [44]. Thus, we considered that carbon ion beam combined with CDDP make the CSCs more easily killed. In addition, we surprisingly found that expressions of angiogenesis-related gene HIF1 $\alpha$ and metastasis-related gene CD26 were significantly suppressed or lost by carbon ion beam combined with CDDP, whereas carbon ion beam alone or X-ray combined with CDDP increased HIF1 $\alpha$, implying that carbon ion beam in combination with CDDP may effectively inhibit tumor angiogenesis and metastasis (Fig. 4d). It has been known that intra-strand lesions, the primary type of DNA damage caused by CDDP are repaired by the nucleotide excision repair (NER) pathway and exquisite sensitivity to CDDP is observed in testicular cancer, which often presents with low expression of NER proteins, such as XPA and ERCC1 [4547]. Although we did not examine NER genes in this study, we found that carbon ion beam combined with CDDP significantly altered expression of NHEJ-related genes (suppressed XRCC4 but enhanced ARTEMIS) and HR-related genes (suppressed 53BP1 and BRAC1 but enhanced RAD51), whereas CDDP alone treatment increased BRCA1 expression, but carbon ion beam, Xray alone or $\mathrm{X}$-ray combined with $\mathrm{CDDP}$ were not affect those of genes (Fig. 4e). In addition, carbon ion beam combined with CDDP significantly increased expression of PTEN and p53, but inhibited cell cycle related Cyclin D1, p21 and p27, whereas CDDP alone treatment significantly increased p21 expression, in comparison, carbon ion beam, X-ray alone or X-ray combined with CDDP were not affect those of genes (Fig. 4f). These findings suggest that carbon ion beam combined with CDDP greatly disrupts DNA repair and cell cycle regulation of BCSCs. Furthermore, cell cycle analyses of MDA-MB-231 and MDA-MB-453 cells by flow cytometry after a carbon ion beam, X-ray alone or in combination with $25 \mu \mathrm{M}$ of CDDP showed that carbon ion beam combined with CDDP more significantly inhibited cell cycle progression (sub-G1 arrest) and induced cell death (apoptosis/necrosis) compared to carbon ion beam alone, X-ray alone, CDDP alone or X-ray combined with CDDP (Fig. 5). All together, carbon ion beam in combination with CDDP appeared to show beneficial effects in inducing various gene expressions in the disruption of TNBCSCs at mRNA levels in vitro, and further investigation for those of genes at protein levels in vitro and in vivo is needed.

In this study, the number of double strand breaks (DSBs) marker $\gamma \mathrm{H} 2 \mathrm{AX}$ foci, formed in CSCs was high at $1 \mathrm{~h}$ after either carbon ion beam or X-ray irradiation alone, and combination with CDDP further increased their number, suggesting that CDDP has sensitization to both X-ray and carbon ion beams. At $24 \mathrm{~h}$ postirradiation the number and size of $\gamma \mathrm{H} 2 \mathrm{AX}$ foci for the carbon ion beam were significantly higher and larger than for X-ray irradiation, revealing the differential repair capacity of the DSBs induced by the high and low LET radiation in CSCs [48-50]. Furthermore, a more larger number of, as well as a larger-sized $\gamma \mathrm{H} 2 \mathrm{AX}$ foci were formed with carbon ion beam combined with CDDP compared to X-ray, carbon ion beam alone or $\mathrm{X}$-ray combined with CDDP, suggesting that higher complexity of clustered DSB was induced by carbon ion beam in combination with CDDP (Fig. 6a, b, c). These results reveal the greater complexity of DSBs induced by high LET radiation combined with chemotherapy, which potentially leads to increased mutagenicity and decreased repairability of the damaged site.

Taken together, because the carbon ion beams have a well-defined range with well-localized energy deposition at the end of the beam path, a unique physical characteristic called "SOBP", and release enormous energy at the end of their range, carbon ion beams therefore induce more cell cycle- and oxygenation-independent, irreparable DNA damage and kill more radioresistant CSCs than photon beams [31, 32], and combination with a DNA-damaging antitumor compound CDDP [51-53] further enhances those of actions based on the present data. This is partially in line with our recent report that 
carbon ion beams combined with gemcitabine, a nucleoside analogue that causes cytotoxicity by inducing DNA replication blocks, efficiently eliminate pancreatic CSCs [54]. Our results showed in this study are the first to show that predominant effects of carbon ion beam in combination with CDDP on TNBC cell killing mainly result from efficient eradication of CSCs rather than non-CSCs.

\section{Conclusions}

In summary, the carbon ion beam combined with CDDP has promising advantages for targeting putative BCSCs because of complex DNA damage, increased apoptosis, autophagy, and subsequent cell death at relatively low doses compared to a carbon ion beam alone. All together, our findings show the potential benefits of a carbon ion beam in combination with chemotherapy to target TNBCSCs.

\section{Additional files}

Additional file 1: Table S1. In vivo limiting dilution assays of sorted MDA-MB-231 and MDA-MB-453 breast cancer cells using surface markers (number of tumors formed/number of injections). (DOCX $16 \mathrm{~kb}$ )

Additional file 2: Figure S1. (PPTX $204 \mathrm{~kb}$ )

\begin{abstract}
Abbreviations
TNBC: Triple-negative breast cancer; HER2: Human epidermal growth factor receptor-2; ER: Estrogen receptor; PR: Progesterone receptor; CSC: Cancer stem-like cell; HIMAC: Heavy ion medical accelerator in Chiba; CDDP: Cisplatin; DMEM: Dulbecco's modified eagle's medium; ANOVA: Analysis of variance; SOBP: Spread-out bragg peak; LET: Linear transfer energy; RBE: Relative biological effectiveness; HIF: Hypoxia inducible factor; NER: Nucleotide excision repair; NHEJ: Non-homologous end joining; HR: Homologous recombination.
\end{abstract}

\section{Competing interests}

The authors declare that they have no competing interests.

\begin{abstract}
Authors' contributions
SS was a primary contributor to study conception, design and experimental implementation. SS performedall the cell line work, FACS sorting data analysis, and wrote the manuscript. GV, BW, and MN provided assistance in performing and analyzing the RT PCR Array experiments. $\mathrm{KK}, \mathrm{YH}$, and $\mathrm{MH}$ provided critical insight about the study design and the clinical meaningful of the experiments. SS, GV and EHK were involved in revising the manuscript, and all authors read and approved the final manuscript.
\end{abstract}

\section{Acknowledgements}

We would like to express our gratefulness to Mr. Yamada T for histopathological slide resection, and HE staining, to Mr. Maeda T, Mr. Takano H, and Dr. Nakajima N for FACS cell sorting and analyze-related technical support. This work was partially supported by Grant-in-Aid for Scientific Research (C) 24591858 from Ministry of Education, Culture, Sports, Science and Technology of Japan (to S. Sai), National Research Foundation of Korea (NRF) No. NRF2014R1A1A3053958 (to E.H. Kim) and by Research Project with Heavy-ion at NIRS-HIMAC

\footnotetext{
Author details

${ }^{1}$ Medical Physics Research Program, Research Center for Charged Particle Therapy, National Institute of Radiological Sciences, 4-9-1 Anagawa Inage-ku, Chiba, Chiba 263-8555, Japan. 'Radiation Risk Reduction Research Program, Research Center for Radiation Protection, National Institute of Radiological Sciences, Chiba, Japan. ${ }^{3}$ Division of Heavy Ion Clinical Research, Korea
}

Institute of Radiological and Medical Sciences, 215-4 Gongneung-dong, Nowon-Gu, Seoul 139-706, South Korea. ${ }^{4}$ Research Center Hospital for Charged Particle Therapy, National Institute of Radiological Sciences, Chiba, Japan. ${ }^{5}$ Department of Breast Oncology, Juntendo University School of Medicine, Tokyo, Japan. 'Department of Breast Oncology, Tokyo Medical University Hachioji Medical Center, Tokyo, Japan.

Received: 21 March 2015 Accepted: 6 August 2015

Published online: 04 September 2015

\section{References}

1. Siegel R, Naishadham D, Jemal A. Cancer statistics, 2012. CA Cancer J Clin. 2012;62:10-29

2. Bhoo-Pathy N, Yip CH, Hartman M, Uiterwaal CS, Devi BC, Peeters PH, et al. Breast cancer research in Asia: adopt or adapt Western knowledge? Eur J Cancer. 2013:49:703-9.

3. Katanoda K, Matsuda T, Matsuda A, Shibata A, Nishino Y, Fujita M, et al. An updated report of the trends in cancer incidence and mortality in Japan. Jpn J Clin Oncol. 2013;43:492-507.

4. Reis-Filho JS, Pusztai L. Gene expression profiling in breast cancer: classification, prognostication, and prediction. Lancet. 2011;378:1812-23.

5. Patani N, Martin LA, Dowsett M. Biomarkers for the clinical management of breast cancer: international perspective. Int J Cancer. 2013;133:1-13.

6. Lehmann BD, Pietenpol JA. Identification and use of biomarkers in treatment strategies for triple-negative breast cancer subtypes. J Pathol. 2014;232:142-50

7. Turner NC, Reis-Filho JS. Tackling the diversity of triple-negative breast cancer. Clin Cancer Res. 2013;19:6380-8.

8. Sioshansi S, Huber KE, Wazer DE. The implications of breast cancer molecular phenotype for radiation oncology. Front Oncol. 2011;1:12.

9. Su Y, Zheng Y, Zheng W, Gu K, Chen Z, Li G, et al. Distinct distribution and prognostic significance of molecular subtypes of breast cancer in Chinese women: a population-based cohort study. BMC Cancer. 2011;11:292.

10. Stevens KN, Vachon CM, Couch FJ. Genetic susceptibility to triple-negative breast cancer. Cancer Res. 2013;73:2025-30.

11. Metzger-Filho O, Tutt A, de Azambuja E, Saini KS, Viale G, Loi S, et al. Dissecting the heterogeneity of triple-negative breast cancer. J Clin Oncol. 2012;30:1879-87

12. Sioshansi S, Ehdaivand S, Cramer C, Lomme MM, Price LL, Wazer DE. Triple negative breast cancer is associated with an increased risk of residual invasive carcinoma after lumpectomy. Cancer. 2012;118:3893-8.

13. Heitz F, Harter P, Lueck HJ, Fissler-Eckhoff A, Lorenz-Salehi F, Scheil-Bertram S, et al. Triple-negative and HER2-overexpressing breast cancers exhibit an elevated risk and an earlier occurrence of cerebral metastases. Eur J Cancer. 2009:45:2792-8.

14. Polyak K. Heterogeneity in breast cancer. J Clin Invest. 2011;121:3786-8.

15. Place AE, Jin Huh S, Polyak K. The microenvironment in breast cancer progression: biology and implications for treatment. Breast Cancer Res. 2011;13:227

16. Higgins MJ, Baselga J. Targeted therapies for breast cancer. J Clin Invest. 2011;121:3797-803.

17. Al-Hajj M, Wicha MS, Benito-Hernandez A, Morrison SJ, Clarke MF. Prospective identification of tumorigenic breast cancer cells. Proc Natl Acad Sci U S A. 2003;100:3983-8.

18. Charafe-Jauffret E, Ginestier C, lovino F, Wicinski J, Cervera N, Finetti P, et al, Breast cancer cell lines contain functional cancer stem cells with metastatic capacity and a distinct molecular signature. Cancer Res. 2009;69:1302-13.

19. Ablett MP, Singh JK, Clarke RB. Stem cells in breast tumours: are they ready for the clinic? Eur J Cancer. 2012;48:2104-16

20. Hambardzumyan D, Squartro M, Holland EC. Radiation resistance and stem-like cells in brain tumors. Cancer Cell. 2006;10:454-6.

21. Rich JN. Cancer stem cells in radiation resistance. Cancer Res. 2007;67:8980-4.

22. Dingli D, Michor F. Successful therapy must eradicate cancer stem cells. Stem Cells. 2006;24:2603-10.

23. Ning X, Shu J, Du Y, Ben Q, Li Z. Therapeutic strategies targeting cancer stem cells. Cancer Biol Ther. 2013;14:295-303.

24. Vermeulen L, de Sousa e Melo F, Richel DJ, Medema JP. The developing cancer stem-cell model: clinical challenges and opportunities. Lancet Oncol. 2012;13:e83-9

25. Tsujii H, Kamada T. A review of update clinical results of carbon ion radiotherapy. Jpn J Clin Oncol. 2012;42:670-85. 
26. Mizoe JE, Hasegawa A, Jingu K, Takagi R, Bessyo H, Morikawa T, et al. Results of carbon ion radiotherapy for head and neck cancer. Radiother Oncol. 2012;103:32-7.

27. Matsumoto K, Imai R, Kamada T, Maruyama K, Tsuji H, Tsujii H, Shioyama Y, Honda $\mathrm{H}$, Isu K: Impact of carbon ion radiotherapy for primary spinal sarcoma. Cancer 2013;119:3496-503.

28. Schulz-Ertner D, Tsujii H. Particle radiation therapy using proton and heavier ion beams. J Clin Oncol. 2007;25:953-64.

29. Allen C, Borak TB, Tsujii H, Nickoloff JA. Heavy charged particle radiobiology: using enhanced biological effectiveness and improved beam focusing to advance cancer therapy. Mutat Res. 2011;711:150-7.

30. Vares G, Cui X, Wang B, Nakajima T, Nenoi M. Generation of breast cancer stem cells by steroid hormones in irradiated human mammary cell lines. PLoS One. 2013;8, e77124.

31. Cui $X$, Oonishi $K$, Tsujii $H$, Yasuda T, Matsumoto $Y$, Furusawa $Y$, et al. Effects of carbon ion beam on putative colon cancer stem cells and its comparison with X-rays. Cancer Res. 2011;71:3676-87.

32. Oonishi K, Cui X, Hirakawa H, Fujimori A, Kamijo T, Yamada S, et al. Different effects of carbon ion beams and X-rays on clonogenic survival and DNA repair in human pancreatic cancer stem-like cells. Radiother Oncol. 2012;105:258-65.

33. Silver DP, Richardson AL, Eklund AC, Wang ZC, Szallasi Z, Li Q, et al. Efficacy of neoadjuvant Cisplatin in triple-negative breast cancer. J Clin Oncol. 2010;28:1145-53.

34. Steg AD, Bevis KS, Katre AA, Ziebarth A, Dobbin ZC, Alvarez RD, et al. Stem cell pathways contribute to clinical chemoresistance in ovarian cancer. Clin Cancer Res. 2012;18:869-81.

35. Phillips TM, McBride WH, Pajonk F. The response of CD24(-/low)/CD44+ breast cancer-initiating cells to radiation. J Natl Cancer Inst. 2006;98:1777-85.

36. Badve S, Nakshatri H. Breast-cancer stem cells—beyond semantics. Lancet Oncol. 2012;13:e43-8.

37. Bao S, Wu Q, McLendon RE, Hao Y, Shi Q, Hjelmeland AB, et al. Glioma stem cells promote radioresistance by preferential activation of the DNA damage response. Nature. 2006;444:756-60.

38. Suzuki M, Kase $Y$, Kanai T, Ando K. Change in radiosensitivity with fractionated-dose irradiation of carbon-ion beams in five different human cell lines. Int J Radiat Oncol Biol Phys. 2000;48:251-8.

39. Peitzsch C, Kurth I, Kunz-Schughart L, Baumann M, Dubrovska A. Discovery of the cancer stem cell related determinants of radioresistance. Radiother Oncol. 2013;108:378-87.

40. Tiwary R, Yu W, Sanders BG, Kline K. alpha-TEA cooperates with chemotherapeutic agents to induce apoptosis of p53 mutant, triplenegative human breast cancer cells via activating p73. Breast Cancer Res. 2011;13:R1.

41. Maycotte P, Aryal S, Cummings CT, Thorburn J, Morgan MJ, Thorburn A. Chloroquine sensitizes breast cancer cells to chemotherapy independent of autophagy. Autophagy. 2012;8:200-12.

42. Debeb BG, Xu W, Mok H, Li L, Robertson F, Ueno NT, et al. Differential radiosensitizing effect of valproic acid in differentiation versus selfrenewal promoting culture conditions. Int J Radiat Oncol Biol Phys. 2010;76:889-95.

43. Pignalosa D, Durante M. Overcoming resistance of cancer stem cells. Lancet Oncol. 2012;13:e187-8.

44. Prabhakaran P, Hassiotou F, Blancafort P, Filgueira L. Cisplatin induces differentiation of breast cancer cells. Front Oncol. 2013;3:134.

45. Usanova S, Piee-Staffa A, Sied U, Thomale J, Schneider A, Kaina B, et al. Cisplatin sensitivity of testis tumour cells is due to deficiency in interstrandcrosslink repair and low ERCC1-XPF expression. Mol Cancer. 2010;9:248.

46. Koberle B, Grimaldi KA, Sunters A, Hartley JA, Kelland LR, Masters JR. DNA repair capacity and cisplatin sensitivity of human testis tumour cells. Int Cancer. 1997;70:551-5

47. Sears CR, Turchi JJ. Complex cisplatin-double strand break (DSB) lesions directly impair cellular non-homologous end-joining (NHEJ) independent of downstream damage response (DDR) pathways. J Biol Chem. 2012;287:24263-72.

48. Yajima H, Fujisawa H, Nakajima NI, Hirakawa H, Jeggo PA, Okayasu R, et al. The complexity of DNA double strand breaks is a critical factor enhancing end-resection. DNA Repair (Amst). 2013;12:936-46.

49. Nakajima NI, Brunton H, Watanabe R, Shrikhande A, Hirayama R, Matsufuji N, et al. Visualisation of gammaH2AX foci caused by heavy ion particle traversal; distinction between core track versus non-track damage PLoS One. 2013;8, e70107.

50. Ibanez IL, Bracalente C, Molinari BL, Palmieri MA, Policastro L, Kreiner AJ, et al. Induction and rejoining of DNA double strand breaks assessed by H2AX phosphorylation in melanoma cells irradiated with proton and lithium beams. Int J Radiat Oncol Biol Phys. 2009;74:1226-35.

51. Boeckman HJ, Trego KS, Turchi JJ. Cisplatin sensitizes cancer cells to ionizing radiation via inhibition of nonhomologous end joining. Mol Cancer Res. 2005;3:277-85

52. Toulany M, Mihatsch J, Holler M, Chaachouay H, Rodemann HP. Cisplatinmediated radiosensitization of non-small cell lung cancer cells is stimulated by ATM inhibition. Radiother Oncol. 2014;111:228-36.

53. Lawrence TS, Blackstock AW, McGinn C. The mechanism of action of radiosensitization of conventional chemotherapeutic agents. Semin Radiat Oncol. 2003;13:13-21.

54. Sai S, Wakai T, Vares G, Yamada S, Kamijo T, Kamada T, et al. Combination of carbon ion beam and gemcitabine causes irreparable DNA damage and death of radioresistant pancreatic cancer stem-like cells in vitro and in vivo. Oncotarget. 2015;6:5517-35.

\section{Submit your next manuscript to BioMed Central and take full advantage of:}

- Convenient online submission

- Thorough peer review

- No space constraints or color figure charges

- Immediate publication on acceptance

- Inclusion in PubMed, CAS, Scopus and Google Scholar

- Research which is freely available for redistribution 